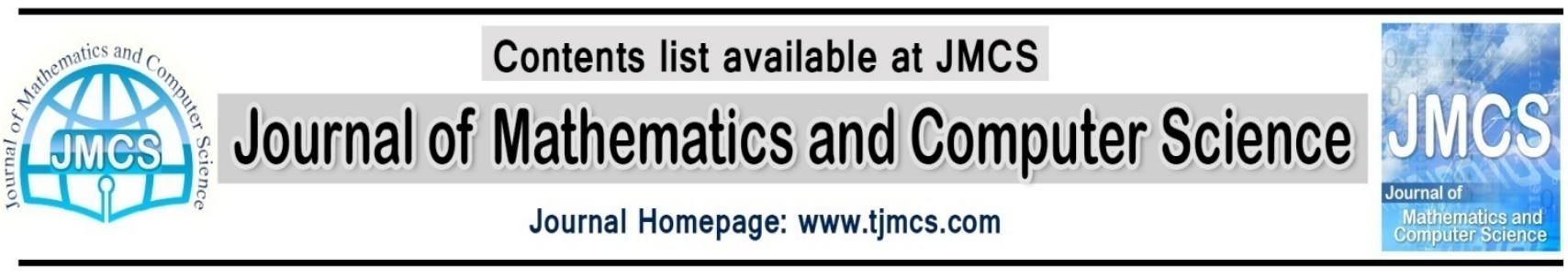

\title{
Use of computer facilities for study of geodesics Schwarzschild Robertson- Walker space-time
}

\author{
Jamal Saffar Ardabili \\ Department of Mathematics, Payame Noor University, P. O. Box. 19395-3697, Tehran, Iran. \\ E-mail: saffar@pnu.ac.ir \\ Mahnaz Ebrahimi
}

Department of Mathematics, University of Mazandaran, Faculty of Sciences, Babolsar, Iran.

Article history:

E-mail: ebrahimi.mahnaz1@gmail.com

Received January 2013

Accepted February 2013

Available online February 2013

\begin{abstract}
We describe the graphical study of geodesic motion on Schwarzshild Robertson-Walker space-time using the symbols and graphical computation facilities of maple platform.
\end{abstract}

Keywords: General relativity, Computer algebra.

\section{Introduction}

Learning to solve the geodesic equations is an integral part of a first course on general relativity. However, most texts on general relativity do not contain a sufficient number of solvable examples that illustrate the behavior of geodesics. This lack is largely due to the fact that the geodesic equations are not easy to solve exactly. The use of Maple in the study of geodesic motion has a long history; see [2] for a list of articles using Maple and GRTensorII package.

The main goal of the article is to bring the geodesic equations in an appropriate form to graphically represent them for visualizing their properties in an easier and striking way. We do this using directly the DEtools package without explicitly solving analytically the equations. This paper illustrates the graphical study of geodesic motion in Schwarzshild Robertson-Wallker spacetime using the symbolic, and graphical computation facilities of the Maple platform + GRTensorII. 


\section{Geodesics in the Schwarshild Robertson-Wallker space-time}

In the standard Schwarzschild $(t, r, \theta, \phi)$, and geometric system of units, the Schwarzschild RobertsonWallker spacetimes are determined by the line element

$$
d s^{2}=-d t^{2}+\frac{s^{2}}{1-k R^{2}} d r^{2}+s^{2} R^{2}\left(d \theta^{2}+\sin \theta^{2} d \phi^{2}\right)
$$

and the coordinates are defined such that $-\infty \leq t \leq+\infty, r \geq 0,0 \leq \theta \leq \pi$ and $0 \leq \phi \leq 2 \pi$.

We can enter this metric directly in this form using ([2], [3]), makeg(...), giving it the name ' "Swr" ':

> restart; grtw(); makeg(Swr):

Makeg 2.0: GRTensor metric/basis entry utility

To quit makeg, type 'exit' at any prompt.

Do you wish to enter a 1) metric [g(dn,dn)],

2) line element [ds],

3) non-holonomic basis [e(1)...e(n)], or

4) NP tetrad [l,n,m,mbar]?

$>$ 2:

Enter coordinates as a LIST (eg. [t,r,theta,phi]):

$>[t, r$, theta, phi]:

Enter the line element using $d[$ coord] to indicate differentials. (for example, $s^{\wedge} 2^{*} R^{\wedge} 2^{*}\left(d[\text { theta }]^{\wedge} 2+\right.$

$\left.\sin (\text { theta })^{\wedge} 2^{*} d[p h i]^{\wedge} 2\right)$ [Type 'exit' to quit makeg]

$\mathrm{ds}^{\wedge} \mathbf{2}=$

$>-(1)^{*} d[t] * d[t]+d[r] * d[r]^{*} s^{\wedge} 2 /\left(1-k^{*} R^{\wedge} 2\right)+s^{\wedge} 2^{*}$

$\mathrm{R}^{\wedge} \mathbf{2}^{*}\left(\mathrm{~d}\left[\right.\right.$ theta] $* d\left[\right.$ theta] $+\left((\sin (\text { theta }))^{\wedge} 2\right) * d[$ phi] $* d[$ phi]);

$>$ makeg completed;

The Christofel symbols in coordinates $(t, r, \theta, \phi)$ are

$$
\begin{gathered}
\Gamma_{r r}^{r}=-\frac{k R}{-1+k R^{2}} \\
\Gamma_{\theta \theta}^{r}=\left(-1+k R^{2}\right) R \sin \theta^{2} \\
\Gamma_{\phi \phi}^{r}=\left(-1+k R^{2}\right) R \\
\Gamma_{r \theta}^{\theta}=\frac{1}{R} \\
\Gamma_{\theta \phi}^{\theta}=\frac{\cos \theta}{\sin \theta} \\
\Gamma_{r \phi}^{\phi}=\frac{1}{R} \\
\Gamma_{\theta \theta}^{\phi}=-\sin \theta \cos \theta .
\end{gathered}
$$

Next we shall define the 4-velocity and 4-acceleration, namely $v^{i}=\frac{d x^{i}}{d \tau} ; a^{i}=\frac{d v^{i}}{d \tau}$ where $\tau$ is the proper time measured in the proper reference frame of the particle. Similar to [2] we shall use an intermediate coordinate set te(tau), er(tau), ph(tau) and th(tau) instead of the coordinates $(t, r, \theta, \phi)$ 
fixed by GrTensorII, till we shall come back to Maple, where there is no possibility of confusion. We do this as a series of GrTensorII definitions; we use some ideas from [1] and [2] in our calculation.

$>\operatorname{grdef}\left(`^{\prime}\left\{\wedge^{\wedge}\right\}:=[\operatorname{diff}(\operatorname{te}(\operatorname{tau}), \operatorname{tau}), \operatorname{diff}(\operatorname{er}(\operatorname{tau}), \operatorname{tau}), \operatorname{diff}(\operatorname{th}(\operatorname{tau}), \operatorname{tau}), \operatorname{diff}(\operatorname{ph}(\operatorname{tau}), \operatorname{tau})]\right.$ ));

$>$ grcalc(v(up)); grdisplay(v(up));

$>$ grdef(’accel\{^i\}:=[diff(te(tau),tau,tau),diff(er(tau),tau,tau)

diff(th(tau),tau,tau),diff(ph(tau),tau,tau)] );

$>$ grcalc(accel(up)); grdisplay(accel(up));

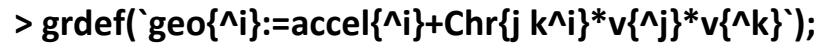

> grcalc(geo(up)); grdisplay(geo(up));

In this way we defin and comput a GrTensorII 4-vector, geo(dn) containing the four geodesic equations. The following commands calculate the next four geodesic equations,

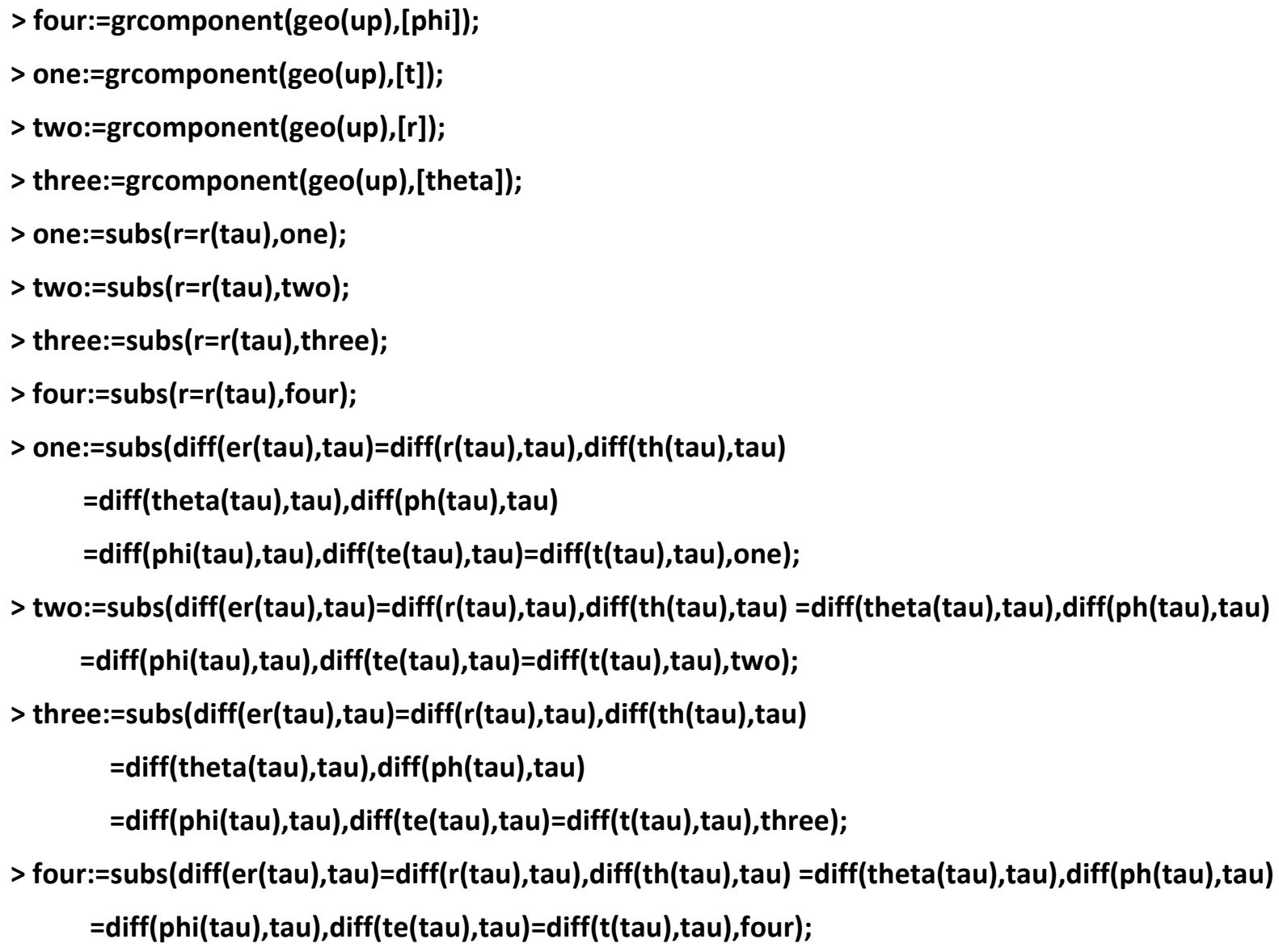

Thus we obtain the next four geodesic equations: 


$$
\begin{gathered}
\frac{d^{2}}{d \tau^{2}} t e(\tau)=0 \\
\frac{\left(1-K R^{2}\right) \frac{d^{2}}{d \tau^{2}} e R(\tau)-K R\left(\frac{d}{d \tau} e R(\tau)\right)^{2}}{-1+K R^{2}} \\
+\frac{\left(1-K R^{2}\right)^{2} R \sin \theta^{2}\left(\frac{d}{d \tau} P h(\tau)\right)^{2}+\left(-1+K R^{2}\right)^{2} R\left(\frac{d}{d \tau} t h(\tau)\right)^{2}}{-1+K R^{2}}=0 \\
\frac{R(\tau) \frac{d^{2}}{d \tau^{2}} t h(\tau)+2\left(\frac{d}{d \tau} e r(\tau)\right)\left(\frac{d}{d \tau} \operatorname{th}(\tau)\right)-R(\tau) \sin \theta \cos \theta\left(\frac{d}{d \tau} p h(\tau)\right)^{2}}{R(\tau)}=0 \\
\frac{R(\tau) \sin \theta \frac{d^{2}}{d \tau^{2}} p h(\tau)+2 \sin \theta\left(\frac{d}{d \tau} e R(\tau)\right)\left(\frac{d}{d \tau} p h(\tau)\right)+2 R(\tau) \cos \theta\left(\frac{d}{d \tau} p h(\tau)\right)\left(\frac{d}{d \tau} \operatorname{th}(\tau)\right)}{R(\tau) \sin \theta}=0
\end{gathered}
$$

We can solve the first equation using the next Maple commands:

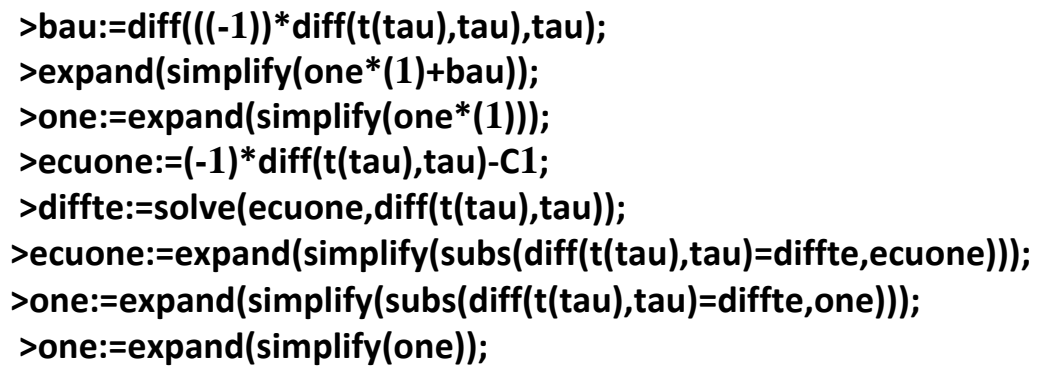

Obtaining for diffte

$$
\frac{d t}{d \tau}=-C_{1}
$$

where $C_{1}$ is a constant.

Now we will solve the four equation as:

$>$ bau2:=diff((R(tau)^2)* $\sin ($ theta(tau) $) \wedge 2 * \operatorname{diff}($ phi(tau),tau),tau);

>expand(simplify $(\operatorname{subs}(\sin ($ theta $($ tau $))=\sin ($ theta $), \cos ($ theta $($ tau $))=$ $\left.\cos (\text { theta), bau2/(R(tau)^2)/sin(theta(tau) })^{\wedge} 2\right)-$ four));

>ecufour:=(R(tau)^2)* $\sin (\text { theta })^{\wedge} 2 * \operatorname{diff}($ phi(tau),tau)-C2;

$>$ diffph:=solve(ecufour,diff(phi(tau),tau));

>ecufour:=expand(simplify(subs(diff(phi(tau),tau)=diffph,ecufour)));

$>$ four:=expand(simplify(subs(diff(phi(tau),tau)= subs(theta=theta(tau),diffph),subs(cos(theta)

$$
=\cos (\text { theta }(\operatorname{tau})), \sin (\text { theta })=\sin (\text { theta(tau)),four })))) \text {; }
$$

$>$ two:=subs(diff(t(tau),tau)=diffte, diff(phi(tau),tau)=diffph,two);

$>$ three:=subs(diff(phi(tau),tau)=diffph,three);

Obtaining for diffph 


$$
\frac{d \phi}{d \tau}=\frac{C 2}{R(\tau)^{2} \sin (\theta)^{2}}
$$

where $C_{2}$ is a constant. To solve the last two remaining equation (two and three) is necessary to fix $\theta=\pi / 2$, thus we have:

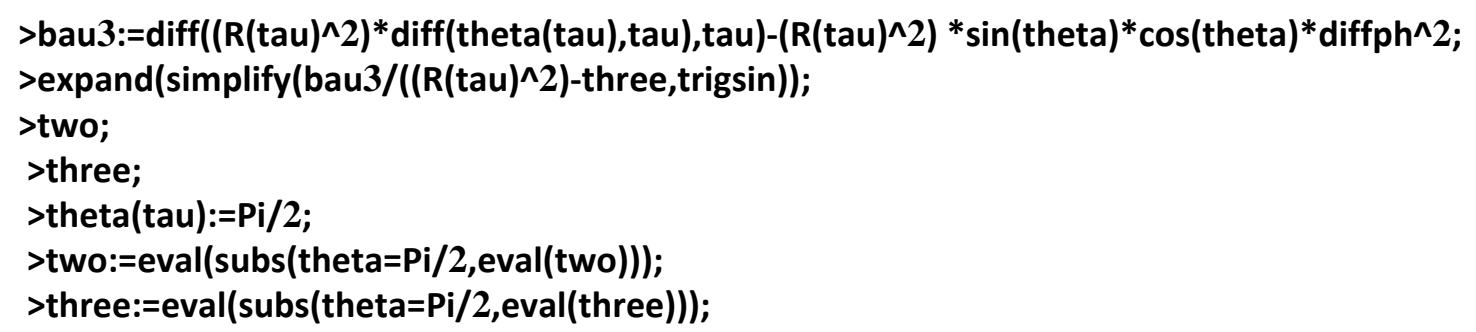

We observe that the second equation (three) is now canceled by the fixing of $\theta$ coordinate. Finally we have the equation $t w o$.

Our purpose is from now on to split the above equation in two different ones, expressing the derivatives for $r$ and $t$ in terms of the angular coordinate $\phi$.

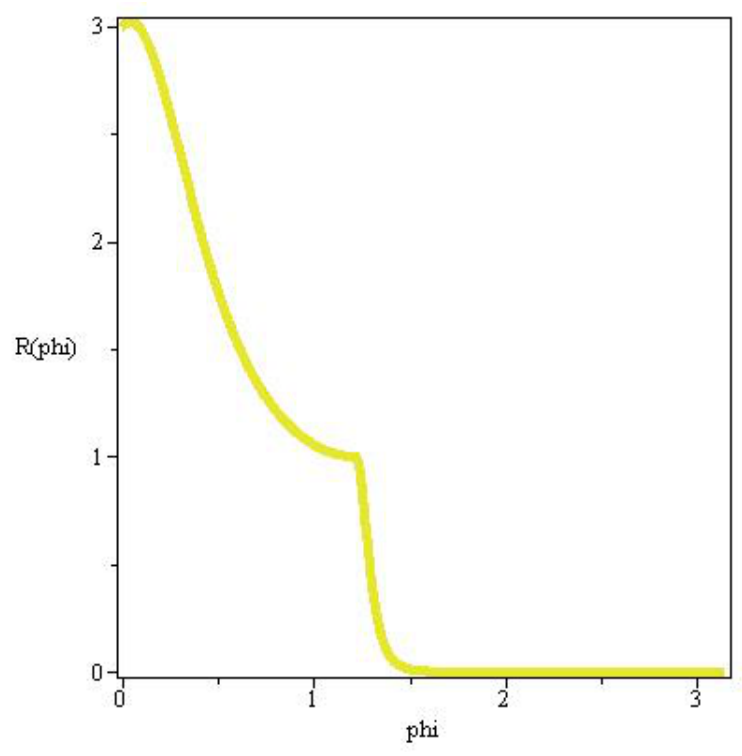

Figure 1:

$>$ aba1:=C2*diff(R(phi),phi)/R(phi)^2;

$>$ aba2:=C2^2/R(phi)^4*(diff(R(phi),phi,phi)-diff(R(phi), phi)^2/R(phi));

$>$ two1:=subs(diff(R(tau),tau,tau)=aba2, $\operatorname{diff}(R($ tau $), \operatorname{tau})=a b a 1, R($ tau $)=R($ phi),two);

>two1:=expand(simplify(two1)); >two2:=diff(t(phi),phi)-C1*r(phi)^3/C2/(R(phi)-1);

Thus we obtain the next two differential equations:

$$
\begin{aligned}
& t w o 1=0 \\
& t w o 2=0 .
\end{aligned}
$$


We used the Maple commands sequence below for numerical integration of the system of equations $(2,3)$. We use $m=1, C_{2}=\pi, C_{1}=1, G=1$.
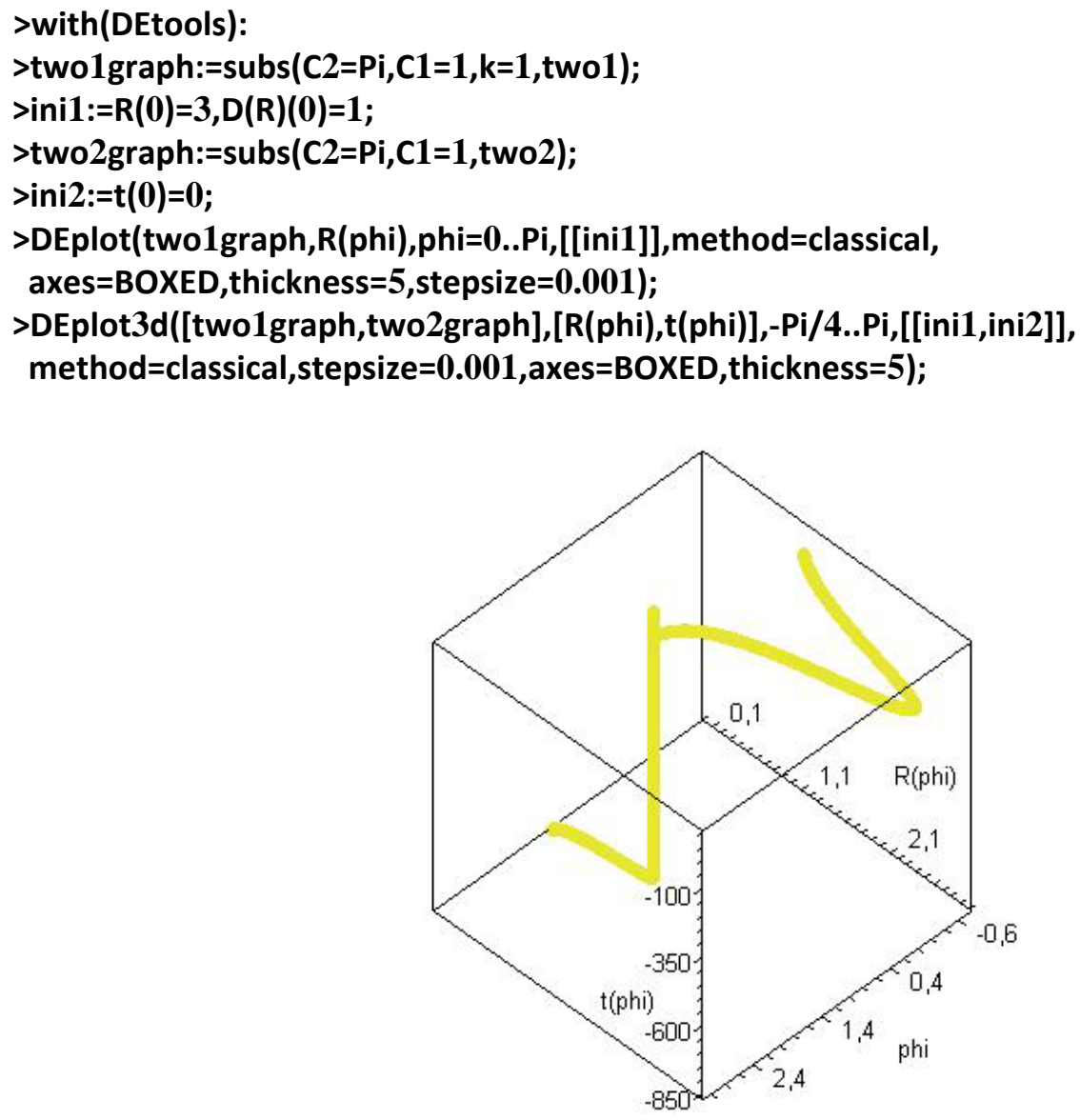

Figure 2:

\section{Conclusion}

We illustrated here the possibility of the study of geodesic motion using the graphical facilities of an integrated (algebraic+graphic) computer platform as Maple. We applied the DEtools package included in several versions of Maple (we mainly used Maple 12) for direct numerical integrations and numerical output plotting of geodesic equations. For this purpose it was necessary to bring the equations in an appropriate form which imposed a special treatment by algebraic computing methods (including tensorial manipulations done in GrTensorII package). This treatment was somehow different of the classical analytical methods used to study the geodesic equations in the literature. The Maple programs and commands sequences we used can be easily adapted for other examples. For instance graphical study of geodesic motion on Schwarzshild de ssiter space-time in [4] and [5] are discussed. 
Jamal Saffar Ardabili, Mahnaz Ebrahimi/ J. Math. Computer Sci. 6 (2013), 139-145

\section{ACKNOWLEDGEMENTS}

The authors gratefully acknowledge the comments by an anonymous referee on the draft of the article.

\section{References}

[1] Dumitru N. Vulcanov. and Valentina D. Vulcanov, The use of Maple platform for the study of geodesic motion on curved spacetimes, IEEE. 0-7695-2740-X/0620.00 @ 2006.

[2] K. M. Heal, M. Hansen and K. Rickard, Maple V Learning Guide for Release 5, Springer Verlag (1997).

[3] P. Musgrave, D. Pollney and K. Lake, GRTensorII: A package for General Relativity, Proceedings of the 6th Canadian Conference in General Relativity and Relativistic Astrophysics, (1996).

[4] M. Rabbani and R. Jamali, Solving nonlinear system of mixed Volterra- Fredholm integral equations by using variational iteration method,TJMCS., 5 (4), 280-287 (2012).

[5] A. Taleshian and M. Ebrahimi, The use of Maple platform for the study of geodesic in the schwarzchild de sitter space-time,TJMCS., 1 (3), 230-237 (2010). 